\title{
Predicting Progression from Mild Cognitive Impairment to Alzheimer's Disease using MRI-based Cortical Features and a Two-State Markov Model
}

\author{
Eleonora Ficiarà ${ }^{a}$, Valentino Crespic ${ }^{c}$, Shruti Prashant Gadewar ${ }^{d}$, Sophia I. Thomopoulos ${ }^{d}$, \\ Joshua Boyd d, Paul M. Thompson ${ }^{d}$, Neda Jahanshad ${ }^{d}$, Fabrizio Pizzagallia \\ and the Alzheimer's Disease Neuroimaging Initiative \\ ${ }^{a}$ Department of Neurosciences, University of Turin, Italy \\ ${ }^{c}$ Information Sciences Institute (ISI), AI Division, University of Southern California, USA \\ ${ }^{d}$ Imaging Genetics Center, Mark and Mary Stevens Neuroimaging and Informatics Institute, \\ Keck School of Medicine of USC, Marina del Rey, CA, USA
}

\begin{abstract}
Magnetic resonance imaging (MRI) has a potential for early diagnosis of individuals at risk for developing Alzheimer's disease (AD). Cognitive performance in healthy elderly people and in those with mild cognitive impairment (MCI) has been associated with measures of cortical gyrification [1] and thickness (CT) [2], yet the extent to which sulcal measures can help to predict AD conversion above and beyond CT measures is not known. Here, we analyzed 721 participants with $\mathrm{MCI}$ from phases 1 and 2 of the Alzheimer's Disease Neuroimaging Initiative, applying a two-state Markov model to study the conversion from $\mathrm{MCI}$ to $\mathrm{AD}$ condition. Our preliminary results suggest that MRI-based cortical features, including sulcal morphometry, may help to predict conversion from MCI to AD.
\end{abstract}

Index Terms - Alzheimer's disease, MRI, Markov model, sulcal morphometry.

\section{INTRODUCTION}

Alzheimer's disease (AD) is the most common type of dementia, causing impaired memory, behavior and cognition with a progressive and irreversible course [3]. AD has long preclinical and prodromal stages and current diagnostic research has focused on identifying early biomarkers that can predict the progression and worsening of dementia.

Together with the core biomarkers of AD (amyloid beta and tau protein), measurements of regional brain volumes from MRI have been shown to be valid biomarkers of both clinical diagnosis and progression of the disease; atrophy rates, for example, are least in patients with unimpaired cognition, increases in $\mathrm{MCI}$ and is the fastest for $\mathrm{AD}$, and also a remarkable change of sulcal widening in the temporal lobe has been observed [4], [5].
Sulcal-based morphometry provides in-depth analyses of the cortical fissures, or folds, as seen on MRI. Interestingly, measures of sulcal morphometry have been associated with neurodegenerative changes in the elderly [2] and found to be genetically correlated [6]. Sulcal changes result from the shrinkage of the adjacent gyri and may also reflect more distal changes that affect the brain's global shape [7].

Recent research has used mathematical modeling to study $\mathrm{AD}$ clinical progression for subjects at different stages, especially MCI - a stage with higher risk of conversion to AD. Analysis of longitudinal biomedical data is often performed using multivariate models [8] and multi-state models, such as a Markov model [9]-[11], which can describe a continuous-time stochastic process that involves transitions among a finite number of states. The state of the Markov process may indicate different severities of a disease.

In this work, we used a two-state Markov model to estimate the effect of patient-related MRI measures (cortical thickness and sulcal width) and to predict how MCI individuals progress to severe dementia. We analyzed the publicly available longitudinal dataset from the Alzheimer's Disease Neuroimaging Initiative (ADNI, http://adni.loni.usc.edu/). Started in 2004, ADNI is an ongoing, longitudinal study across 58 North American sites, designed to unravel biomarkers for the early detection and tracking of $\mathrm{AD}$.

\section{METHODS}

\subsection{Dataset}

We analyzed longitudinal data from the first two phases of ADNI (ADNI1 and ADNI2) including records from 721 individuals affected by MCI. Individual records include a 
person's age, sex, brain MRI, Mini-Mental State Examination (MMSE), Clinical Dementia Rating-Sum of Boxes score (CDR-SB), and clinical diagnosis (MCI or AD). All individuals analyzed in this study were first diagnosed with MCI at baseline; the follow-up scan was performed approximately two years later, when their condition was re-assessed to establish whether they had transitioned to AD. We ran FreeSurfer 5.3 to process MRI data and extract the cortical thickness from 68 regions of interest. Next, we used the ENIGMA-Sulci protocol (http://enigma.ini.usc.edu) to identify and estimate the sulcal width [6], [12], [13] for 123 sulci across the whole brain, based on BrainVISA suite [14]. Finally, we applied a Relief-based feature selection algorithm (available in the $R$ package, CORElearn), to rank raw features according to their classification relevance [15] and select the top five, hereafter also referred to as covariates. This process produced two sets of five covariates (five sulci and five cortical regions).

\subsection{Mathematical Model}

For each of the two groups of five features (Section 2.1) we can formalize the respective dataset as a set of $N=721$ sequences of two labeled vectors:

$$
D=\left\{\left(\left(y_{i, 1}, l_{i, 1}\right),\left(y_{i, 2}, l_{i, 2}\right)\right) \mid i=1,2, \ldots, N\right\},
$$

with $y_{i, j} \in R^{5}$ and $l_{i, j} \in\{M C I, A D\}, j=1,2$. Thus, $y_{i, j}$ is the $j^{\text {th }}$ vector of 5 covariates extracted from the $j^{\text {th }}$ examination of patient $i$ and $l_{i, j}$ is the ground truth diagnosis based on medical examination by experts during the visit. As each individual is examined twice, there is a sequence of two labeled vectors for each individual in $D$. Let $S_{t}$ and $y_{t}$ be, respectively, the medical condition - MCI or AD - of an individual at time $t$ and his/her corresponding vector of observed covariates. This means that at time $t$ the individual is visited and brain MRI data taken; s/he then receives diagnosis $S_{t}$ while vector $y_{t}$ is extracted from the MRI data.

Our approach consists of a) using part of $D$ to train a stochastic model, $\lambda$, that would represent the conditional probability distributions $P_{\lambda}\left(S_{t+\Delta t} \mid S_{t}, y_{t}\right)$, and b) using $\lambda$ to predict whether a test patient will transition to $\mathrm{AD}$ within the next two years, given the outcome of the first visit. Formally, we compute $P_{\lambda}\left(S_{t+\Delta t}=j \mid S_{t}=i, y_{t}\right)$, $i, j \in\{M C I, A D\}$, for $\Delta t=2$ years. To be clear, $P_{\lambda}\left(S_{t+2 y r}=A D \mid S_{t}=M C I, y_{t}\right)$ is the conditional probability that a test individual will transition to $\mathrm{AD}$ by the second visit, namely within two years, given that the vector of covariates $y_{t}$ was observed at his/her first visit. Following the approach in [10] we considered a continuous homogeneous Markov chain with covariates. Accordingly, let $P_{i, j}(t, t+\Delta t)=P\left(S_{t+\Delta t}=j \mid S_{t}=i, y_{t}\right)$ then

$$
P(t, t+\Delta t)=\exp (\Delta t Q(t)) \approx I+\Delta t Q(t),
$$

with $I$ the identity matrix, $P=\left[P_{i, j}\right]_{i, j}, Q=\left[Q_{i, j}\right]_{i, j}$, and

$$
Q_{i, j}(t)=Q_{0, i, j} \exp \left(\beta_{i, j}{ }^{T} y_{t}\right), i \neq j \text {, and } Q_{i, i}(t)=-\sum_{j: i \neq j} Q_{i, j}(t) \text {. }
$$

We assumed that the probability of reverting to MCI from the AD condition was negligible, implying that $P_{\lambda}\left(S_{t+\Delta t}=M C I \mid S_{t}=A D\right)=0$, and that the conversion from MCI to cognitively healthy was negligible. The homogeneity assumption implies that the transition rates, $Q_{0, i, j}$, are constant. We used $R$ code from [10], with proper modifications, to estimate all the parameters of the model, $Q_{0, i, j}$ and $\beta_{i, j}$, for all $i, j$, and compute survival probabilities $P_{\lambda}\left(S_{t+\Delta t}=M C I \mid S_{t}=M C I, y_{t}\right)$.

Finally, we built the actual predictor deciding whether a subject will transition to the AD state by applying a trained logistic regression to the vector composed of all the estimated probabilities and rates, $P_{i, j}$ and $Q_{i, j}$. We performed stratified 10-fold cross-validation analysis (one cycle) computing mean and standard deviations of the Areas Under Curve (AUC) and accuracy.

\section{RESULTS}

The dataset includes $721 \mathrm{MCI}$ individuals, 533 of whom were categorized as having stable MCI (sMCI) (age $=73.0 \pm$ 7.5 years, $($ Male $/$ Female $)=(319 / 214))$ and $188 \mathrm{MCI}$ individuals who later converted to AD (MCI-AD) (age=73.6 \pm 7.3 years, $($ Male $/$ Female $)=(112 / 76))$. There was no significant difference in age distribution between sMCI and MCI-AD. At baseline, MMSE of the MCI-AD group (26.7 $\pm 1.8)$ showed significant differences $(p<0.001)$ with respect to $\mathrm{sMCI}(27.9 \pm 1.7)$. The CDR-SB of group MCI-AD $(2.1$ $\pm 1.0)$ was also significantly different $(p<0.001)$ than that of the sMCI group $(1.4 \pm 0.8)$.

Figure 1 depicts the two-state Markov chain based on the number of transitions from $\mathrm{MCI}$ to $\mathrm{AD}$ and considering $\mathrm{AD}$ as the absorbing state.

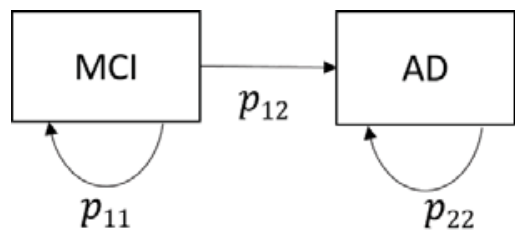

Figure 1. Two-state Markov model for the conversion of MCI patients to the AD state. Arrows indicate possible transitions between the two states. $p_{12}$ (MCI-AD/Total patients) is the transition probability from $\mathrm{MCI}$ to $\mathrm{AD}$, and $p_{11}$ (sMCI/Total patients) is the transition probability of remaining in the MCI condition. Values of $p_{12}, p_{11}$ and $p_{22}$ are set to $0.26,0.74$, and 1 respectively. 
We explored different homogeneous continuous-time Markov models to investigate the progression of dementia, progressively incorporating covariates into transition rates, as explained in Section 2.2.

\subsection{Model without covariates}

First, we built a baseline model without covariates. The transition rate - describing the speed of transition probability with respect to time, from MCI to $\mathrm{AD}$ - was estimated to be

0.34 with $95 \%$ confidence interval $(0.29,0.39)$. Table 1 reports the resulting probability (as inferred from the estimated model rates) of a subject transitioning from the $\mathrm{MCI}$ to $\mathrm{AD}$ state for $\Delta t=2$ years and $\Delta t=4$ years.

According to the baseline model, a subject in the MCI state has $29 \%$ chance of transitioning to the AD state within two years and this probability increases over four years (to $49 \%$ ). The probability of not entering state $\mathrm{AD}$ can be evaluated from the survival curve (Figure 2).

Table 1. 95\% confidence interval estimates of transition probabilities for the baseline model (no covariates).

\begin{tabular}{ccc}
\hline Transition & $\begin{array}{c}\text { Probability } \\
\text { (Year 2) }\end{array}$ & $\begin{array}{c}\text { Probability } \\
\text { (Year 4) }\end{array}$ \\
\hline MCI-MCI & $0.72(0.68,0.75)$ & $0.51(0.46,0.56)$ \\
\hline MCI-AD & $0.29(0.25,0.33)$ & $0.49(0.44,0.54)$
\end{tabular}

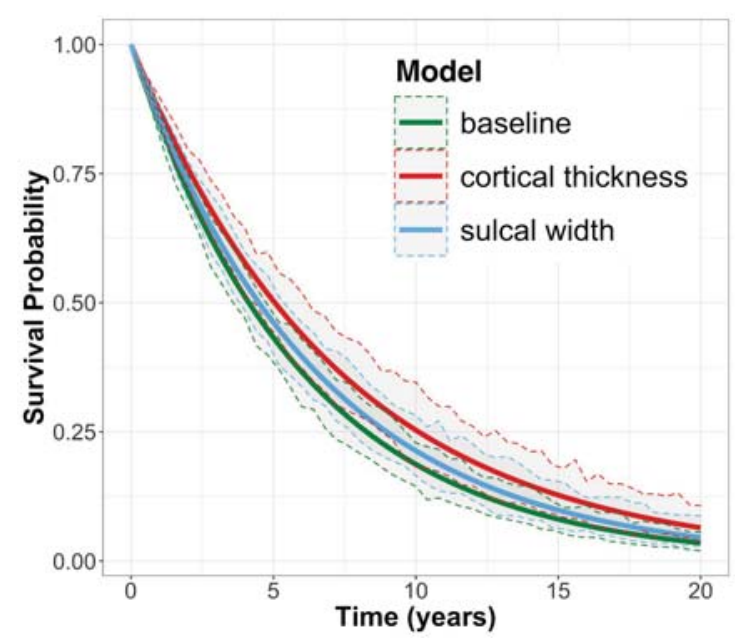

Figure 2. Survival curves for the baseline model (no covariates) and after including cortical thickness (red) and sulcal width (blue) as covariates. The solid line is the fitted curve describing the likelihood of conversion from MCI to $\mathrm{AD}$, for time intervals up to 20 years, and dotted lines indicate the $95 \%$ confidence band for the corresponding curve.

\subsection{Model with covariates}

It is commonly assumed that age is a factor contributing to the acceleration of the MCI-AD transition [16]. However, adding age to the baseline model as a covariate did not yield significant benefits, as confirmed by the likelihood ratio test (LRT) between the baseline model (null hypothesis) and the augmented model $(p=0.12)$. We then considered the top 5 features extracted and selected from MRI measurements of sulcal width and cortical thickness as explained in Section 2.1:

- Sulcal Width:

S.O.T.lat.ant._avg+S.O.T.lat.med._avg+INSULA_avg+

F.C.L.r.asc._avg+ S.T.s._avg (see Figure 3 and

http://brainvisa.info [14] for the meanings of these sulcal labels; the term_avg refers to the mean of right and left hemisphere measurements).

- Cortical Thickness:

RThickness + LThickness $+\mathrm{R}$ fusiform thickavg $+\mathrm{R}$ infer iortemporal_thickavg $+\mathrm{L}$ _fusiform_thickavg (see FreeSurfer's Desikan-Killiany Atlas).

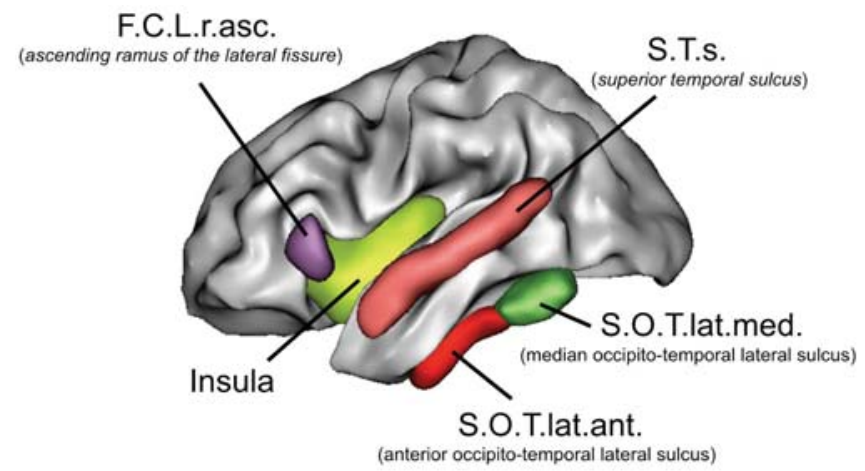

Figure 3. Representation of the five sulci selected as covariates of the model.

Augmenting the baseline model with sulcal widths, as explained in Section 2.2, caused the transition rate estimate from $\mathrm{MCI}$ to $\mathrm{AD}$ to change to 0.31 with $95 \%$ confidence interval $(0.25,0.37)$. The LRT now revealed a difference between the baseline and augmented models $(p<0.001$, $-2 \log L R=130.2$ ). Table 2 reports the resulting probability of a subject transitioning from state $\mathrm{MCI}$ to state $\mathrm{AD}$ for $\Delta t=2$ years and $\Delta t=4$ years. 
According to this second model, a subject in the MCI state has $27 \%$ chance of transitioning to the $\mathrm{AD}$ state within two years and this probability increases over four years (46\%). The probability of not entering the AD state can be evaluated from the survival curve (Figure 2).

Table 2. 95\% confidence interval estimates of transition probabilities for the model with sulcal widths as covariates.

\begin{tabular}{ccc}
\hline Transition & $\begin{array}{c}\text { Probability } \\
\text { (Year 2) }\end{array}$ & $\begin{array}{c}\text { Probability } \\
\text { (Year 4) }\end{array}$ \\
\hline MCI-MCI & $0.73(0.69,0.78)$ & $0.54(0.48,0.61)$ \\
\hline MCI-AD & $0.27(0.22,0.31)$ & $0.46(0.40,0.52)$
\end{tabular}

We built a third model by augmenting the baseline model with cortical thickness measurements as covariates. In this case, the transition rate estimate from MCI to $\mathrm{AD}$ changed to 0.27 with $95 \%$ confidence interval $(0.23,0.33)$. Also in this case, the LRT revealed a difference between the baseline model and the augmented model $(p<0.001$, $-2 \log L R=100.0$ ). Table 3 reports the new transition probabilities after incorporating cortical thicknesses as covariates. Figure 2 shows the corresponding survival curve.

Table 3. $95 \%$ confidence interval estimates of transition probabilities for the model with cortical thicknesses as covariates.

\begin{tabular}{ccc}
\hline Transition & $\begin{array}{c}\text { Probability } \\
(\text { Year 2) }\end{array}$ & $\begin{array}{c}\text { Probability } \\
\text { (Year 4) }\end{array}$ \\
\hline MCI-MCI & $0.76(0.72,0.80)$ & $0.58(0.52,0.64)$ \\
\hline MCI-AD & $0.24(0.20,0.28)$ & $0.42(0.36,0.49)$ \\
\hline
\end{tabular}

Finally, we used the estimated transition probabilities and rates of our models to train a logistic regression model. The test set was used to obtain the predicted diagnostic state (stable MCI or converter to AD condition), showing higher AUC and accuracy for the model incorporating covariates from MRI-based features from sulcal width $(\mathrm{AUC}=0.71$ \pm 0.09 ; Accuracy $=0.72 \pm 0.04)$ and cortical thickness $(\mathrm{AUC}=0.78 \pm 0.08 ;$ Accuracy $=0.75 \pm 0.02) \quad$ compared to the baseline model $(\mathrm{AUC}=0.56 \pm 0.10 ;$ Accuracy $=0.65$ $\pm 0.08)$.

\section{DISCUSSION AND CONCLUSIONS}

In this work, we applied a two-state Markov model to investigate the progression of dementia, considering the conversion from MCI to AD. The results of the model are in accordance with the likelihood of progression from MCI to other forms of dementia reported in the literature [16], having MCI individuals approximately $15 \%$ transition rate annually to convert to AD condition [17].

In addition, we explored the potential role of MRI-based measurements (sulci width and cortical thickness). The widening of cortical sulcal shape has been widely accepted as a neuroimaging marker of age- or disease-related brain atrophy [1], [7], [18], although few studies have used quantitative sulcal morphometry to assist predictive modeling. Markov models have been considered good models to measure the dynamic association between brain structure changes and progressive stages of disease, exploiting longitudinal brain image data [19].

In our work, we included measurements of sulci width and cortical thickness as covariates of Markov models, showing that they can affect the transition rate and transition probability of the conversion MCI to AD. The transition rate and transition probabilities can be used to make predictions of the disease state over time. The discovery of new predictive biomarkers affecting the worsening of the disease gained interest for an early detection of $\mathrm{AD}$ and consequently a better tuning of treatments in different stages of the disease. Our preliminary results suggest that MRI-based cortical features can help to improve the prediction of the conversion from MCI to AD. A thorough comparison of other MRI-based features, such as volumetric measures, from different brain regions, tested with different classifiers, will be investigated in future works.

Compliance with Ethical Standards: This study was conducted retrospectively using anonymized, de-identified human subjects data made available for open access at http://adni.loni.usc.edu/. Additional ethical approval was not required, as confirmed by the license for using the open access data.

\section{ACKNOWLEDGMENTS}

This research was supported in part by the National Institutes of Health (NIH) under grants R01AG059874, RF1AG051710, and P41EB015922. No conflict of interest. 


\section{REFERENCES}

[1] K. Cai et al., "Identification of Early-Stage Alzheimer's Disease Using Sulcal Morphology and Other Common Neuroimaging Indices," PLoS One, vol. 12, no. 1, p. e0170875, Jan. 2017.

[2] J. P. Lerch, J. C. Pruessner, A. Zijdenbos, H. Hampel, S. J. Teipel, and A. C. Evans, "Focal decline of cortical thickness in Alzheimer's disease identified by computational neuroanatomy," Cereb. Cortex, vol. 15, no. 7, pp. 995-1001, Jul. 2005.

[3] A. D. International, "World Alzheimer report 2019: attitudes to dementia." Alzheimer's Disease International London, UK, 2019.

[4] K. Im, J.-M. Lee, S. W. Seo, S. Hyung Kim, S. I. Kim, and D. L. Na, "Sulcal morphology changes and their relationship with cortical thickness and gyral white matter volume in mild cognitive impairment and Alzheimer's disease," Neuroimage, vol. 43, no. 1, pp. 103-113, Oct. 2008.

[5] G. B. Frisoni, N. C. Fox, C. R. Jack, P. Scheltens, and P. M. Thompson, "The clinical use of structural MRI in Alzheimer disease," Nat. Rev. Neurol., vol. 6, no. 2, pp. 67-77, Feb. 2010.

[6] F. Pizzagalli et al., "The reliability and heritability of cortical folds and their genetic correlations across hemispheres," Commun Biol, vol. 3, no. 1, p. 510, Sep. 2020.

[7] K. Jin, T. Zhang, M. Shaw, P. Sachdev, and N. Cherbuin, "Relationship Between Sulcal Characteristics and Brain Aging," Front. Aging Neurosci., vol. 10, p. 339, Nov. 2018.

[8] K. Liu, K. Chen, L. Yao, and X. Guo, "Prediction of Mild Cognitive Impairment Conversion Using a Combination of Independent Component Analysis and the Cox Model," Front. Hum. Neurosci., vol. 11, p. 33, Feb. 2017.

[9] A. Martino, G. Guatteri, and A. M. Paganoni, "Multivariate Hidden Markov Models for disease progression," Statistical Analysis and Data Mining: The ASA Data Science Journal, vol. 13, no. 5. pp. 499-507, 2020, doi: 10.1002/sam.11479.

[10] L. Zhang et al., "Analysis of conversion of Alzheimer's disease using a multi-state Markov model," Stat. Methods Med. Res., vol. 28, no. 9, pp. 2801-2819, Sep. 2019.

[11] C. Hadjichrysanthou, A. K. Ower, F. de Wolf, R. M. Anderson, and for the Alzheimer's Disease Neuroimaging Initiative, "The development of a stochastic mathematical model of Alzheimer's disease to help improve the design of clinical trials of potential treatments," PLOS ONE, vol. 13, no. 1. p. e0190615, 2018, doi: 10.1371/journal.pone.0190615.

[12]F. Pizzagalli, G. Auzias, and P. Kochunov, "The core genetic network underlying sulcal morphometry," 12th International, 2017, [Online]. Available: https://www.spiedigitallibrary.org/conference-proceedi ngs-of-spie/10160/101600C/The-core-genetic-networkunderlying-sulcal-morphometry/10.1117/12.2256959.sh ort.

[13] M. Dojat, F. Pizzagalli, and J.-M. Hupé, "Magnetic resonance imaging does not reveal structural alterations in the brain of grapheme-color synesthetes," PLoS One, vol. 13, no. 4, p. e0194422, Apr. 2018.

[14] M. Perrot, D. Rivière, and J.-F. Mangin, "Cortical sulci recognition and spatial normalization," Med. Image Anal., vol. 15, no. 4, pp. 529-550, Aug. 2011.

[15]R. J. Urbanowicz, M. Meeker, W. La Cava, R. S. Olson, and J. H. Moore, "Relief-based feature selection: Introduction and review," J. Biomed. Inform., vol. 85, pp. 189-203, Sep. 2018.

[16] N. L. Campbell, F. Unverzagt, M. A. LaMantia, B. A. Khan, and M. A. Boustani, "Risk Factors for the Progression of Mild Cognitive Impairment to Dementia," Clinics in Geriatric Medicine, vol. 29, no. 4. pp. 873-893, 2013, doi: 10.1016/j.cger.2013.07.009.

[17] R. C. Petersen, J. C. Stevens, M. Ganguli, E. G. Tangalos, J. L. Cummings, and S. T. DeKosky, "Practice parameter: Early detection of dementia: Mild cognitive impairment (an evidence-based review): Report of the Quality Standards Subcommittee of the American Academy of Neurology," Neurology, vol. 56, no. 9. pp. 1133-1142, 2001, doi: 10.1212/wnl.56.9.1133.

[18] P. Kochunov et al., "Age-related morphology trends of cortical sulci," Hum. Brain Mapp., vol. 26, no. 3, pp. 210-220, 2005.

[19] Y. Wang, S. M. Resnick, C. Davatzikos, Baltimore Longitudinal Study of Aging, and Alzheimer's Disease Neuroimaging Initiative, "Analysis of spatio-temporal brain imaging patterns by Hidden Markov Models and serial MRI images," Hum. Brain Mapp., vol. 35, no. 9 , pp. 4777-4794, Sep. 2014. 